\title{
Achados Ultra-Sonográficos em Pacientes com Ameaça de Abortamento no Primeiro Trimestre da Gestação
}

\author{
Ultrasound Findings in First-trimester Threatened Abortion \\ Luiz Carlos Watanabe, Maria de Lourdes Brizot, Pedro Paulo Pereira, \\ Samir Abdalla Mustafá, Seizo Miyadahira, Marcelo Zugaib
}

\begin{abstract}
RESUMO
Objetivo: avaliar os achados ultra-sonográficos nas gestantes com ameaça de abortamento no primeiro trimestre da gestação.

Métodos: exames de ultra-som obstétrico transabdominal e transvaginal foram realizados nas pacientes com sangramento vaginal e teste de gravidez positivo. Foram incluidas as gestantes entre 6 a 14 semanas de gestação pela data da última menstruação ou pelo exame ultra-sonográfico, que apresentavam colo do útero impérvio ao exame clínico. Foram excluídas as gestações múltiplas ou aquelas que realizaram tentativa de abortamento por meio de drogas ou manipulação.

Resultados: em 132 dos 247 casos (53.4\%) foi diagnosticada gestação viável e em 46.6\% (115/247), gestação inviável. Abortamento incompleto foi identificado em 19\% (47/247), abortamento completo em 8,5\% (21/247), abortamento retido em 7,7\% (19/247), gestação anembrionada em 6,1\% (15/247), gestação ectópica em 4,5\% (11/247) e mola hidatiforme em $0,8 \%$ (2/247).

Conclusão: aproximadamente metade (46,6\%) das gestações com ameaça de abortamento no primeiro trimestre apresentaram diagnóstico de inviabilidade da gestação. O exame ultrasonográfico pode ajudar a definir esta condição e a conduta obstétrica.
\end{abstract}

PALAVRAS-CHAVE: Ameaça de Abortamento. Sangramento Primeiro Trimestre da Gestação. Ultrassonografia. Mola hidatiforme.

\section{Introdução}

A ameaça de abortamento é definida como a presença de sangramento vaginal com colo do útero impérvio ao exame clínico, sendo complicação relativamente freqüente que ocorre em cerca de 16 a $25 \%$ das gestações reconhecidas ${ }^{1-3}$. Apesar da possibilidade deste sangramento estar relacionado a causas vaginais ou cervicais, o abortamento constitui a principal preocupação do casal, levando na maioria das vezes à procura do seu médico.

Após episódio de sangramento vaginal, a

Clínica Obstétrica do Hospital das Clínicas da Faculdade de Medicina da Universidade de São Paulo

Setor de Medicina Fetal

Correspondência:

Maria de Lourdes Brizot

Clínica Obstétrica da Faculdade de Medicina da USP

Av. Dr. Enéas Carvalho de Aguiar, 2555 - 10 andar - ICHC 05403-000 - São Paulo - SP inviabilidade da gestação é observada em cerca de $50 \%$ dos casos a despeito das medidas terapêuticas adotadas ${ }^{2,4,5}$. O exame ultra-sonográfico tem sido empregado como método diagnóstico capaz de diferenciar as gestações viáveis das inviáveis. Anteriormente ao advento desta tecnologia, adotava-se para todos os casos conduta expectante, aguardando-se a possibilidade da ocorrência clínica do abortamento espontâneo para se intervir ${ }^{5}$.

Quando, por meio do exame ultra-sonográfico, é identificada a inviabilidade da gestação, várias são as possibilidades diagnósticas: abortamento completo, abortamento incompleto, abortamento retido, gestação anembrionada, gestação ectópica e doença trofoblástica gestacional. A identificação destes casos permite conduta atuante por parte do obstetra, reduzindo os riscos de sangramento e infecção ${ }^{5}$. Nos casos em que é diagnosti- 
cado feto vivo, a gestação em geral apresenta bom prognóstico, com risco de perda subseqüente em torno de 10 a $20 \%{ }^{6-9}$.

Com a finalidade de observar os achados ultra-sonográficos nos casos com ameaça de abortamento e a importância deste exame associado ao quadro clínico, desenvolvemos este estudo em gestantes no primeiro trimestre de gravidez, que procuraram o Hospital das Clínicas da FMUSP apresentando ameaça de abortamento.

\section{Pacientes e Métodos}

Este trabalho foi realizado no período de junho de 1998 a fevereiro de 1999 e consistiu na avaliação ultra-sonográfica das gestantes que procuraram auxílio no Pronto-Socorro ou Ambulatório de Obstetrícia do Hospital das Clínicas da FMUSP, com queixa de sangramento vaginal. Inicialmente as pacientes foram submetidas a consulta médica com anamnese e exame físico. Exame laboratorial de $\beta$-hCG foi realizado para comprovação da gravidez. Nas gestantes que apresentaram quadro clínico de ameaça de abortamento (sangramento vaginal e colo do útero impérvio ao exame fisico) foi indicado o exame ultra-sonográfico para avaliação da viabilidade da gestação. Os exames de ultra-sonografia foram realizados no Setor de Medicina Fetal da Clínica Obstétrica por via transabdominal e transvaginal. Foram utilizados rotineiramente transdutor setorial convexo de $3.5 \mathrm{MHz}$ para a via abdominal e transdutor convexo transvaginal de $6.0 \mathrm{MHz}$. A idade gestacional foi estabelecida pela data da última menstruação e corrigida pela biometria do exame ultra-sonográfico quando a diferença entre ambas foi superior a sete dias. Foram incluídos os casos com idade gestacional entre seis e quatorze semanas. Foram excluídas as gestações múltiplas e as pacientes que realizaram tentativa de abortamento por uso de medicação ou manipulação.

Os casos foram agrupados em gestações viáveis (na presença de batimento cardiaco embrionário ou fetal) e inviáveis (abortamento completo, abortamento incompleto, abortamento retido, gestação anembrionada, mola hidatiforme e gravidez ectópica).

\section{Resultados}

Foram incluídas nesta avaliação 247 gestantes com ameaça de abortamento. As gestações viáveis totalizaram $53,4 \%(132 / 247)$ e as gestações inviáveis 46,6\% $(115 / 247)$.

A variação da idade materna foi de 12 a 45 anos. A idade materna média para as gestações viáveis foi de 26,8 anos com mediana de 26 anos. A idade materna média para as gestações inviáveis foi de 26,9 anos com mediana de 26 anos. As gestantes com idade até 30 anos corresponderam a $66,8 \%(165 / 247)$ dos casos, entre 30 e 35 anos corresponderam a $22,3 \%(55 / 247)$ e com mais de 35 anos a 10,9\% (27/247). Quando comparado o percentual de gestações inviáveis com a idade materna, verificamos que na faixa etária até 30 anos, 46,6\% (77/165) das gestações eram inviáveis, entre 30 a 35 anos, $45 \%(25 / 55)$, e acima de 35 anos, 48\% (13/27).

A duração do sangramento vaginal apresentou variação de um a quarenta dias, com média de 5,7 dias e mediana de três dias. Quando o tempo de sangramento foi comparado com a viabilidade da gestação, verificamos que as gestações viáveis apresentaram mediana de três dias e as gestações inviáveis, mediana de cinco dias.

A idade gestacional média foi de 9,9 semanas, com variação de seis a quatorze semanas de gestação. Quando o sangramento vaginal ocorreu entre seis e nove semanas de gestação, foi identificada inviabilidade em $44,2 \%$ dos casos. Entre 10 e 14 semanas a inviabilidade foi de $39,8 \%$.

$\mathrm{O}$ antecedente de abortamento em gestação prévia foi identificado em 40,9\% (54/132) das gestações viáveis e em 27,8\% (32/115) das gestações inviáveis.

$\mathrm{Na}$ Tabela 1 apresentamos os grupos com gestações viáveis e inviáveis em relação ao número de pacientes, tempo de sangramento e idade materna.

Tabela 1 - Comparação entre os grupos de gestações viáveis e inviáveis com relação à idade materna e tempo de sangramento.

\begin{tabular}{lcccccc}
\hline & & \multicolumn{3}{c}{ Idade Materna } & \multicolumn{2}{c}{ TS } \\
Grupos & $\mathbf{n}$ & $\%$ & (Média) & Média & Mediana \\
\hline Gestação viável & 132 & 53,4 & 26,9 anos & 5,6 & 3 \\
Gestação inviável & 115 & 46,6 & 26,8 anos & 5,8 & 5
\end{tabular}

TS - Tempo de sangramento (dias).

Na Tabela 2 estão os casos de gestações inviáveis distribuídas de acordo com o achado ultra-sonográfico, especificando a idade materna média e o tempo de sangramento de cada entidade diagnóstica. Observam-se valores similares de idade materna e tempo de sangramento entre todos; exceção ocorreu nos casos de mola hidatiforme, que apresentaram idade materna e tempo de sangramento menores. Entretanto, este grupo foi constituído por apenas dois casos. 
Tabela 2 - Distribuição das gestações inviáveis $(n=115)$, de acordo com 0 achado ultrasonográfico, idade materna e tempo de sangramento.

\begin{tabular}{lcccc}
\hline Grupos & $\mathbf{n}$ & $\%$ & $\begin{array}{c}\text { Idade Materna } \\
\text { (Média) }\end{array}$ & $\begin{array}{c}\text { TS } \\
\text { Média }\end{array}$ \\
\hline Abortamento incompleto & 47 & 19 & 26,8 & 5,7 \\
Abortamento Completo & 21 & 8,5 & 26,9 & 5,7 \\
Abortamento retido & 19 & 7,7 & 26,8 & 5,8 \\
Gestação anembrionada & 15 & 6,1 & 26,6 & 5,9 \\
Gravidez ectópica & 11 & 4,5 & 26,7 & 5,9 \\
Mola hidatiforme & 2 & 0,8 & 20 & 1 \\
\hline
\end{tabular}

TS - Tempo de sangramento (dias).

\section{Discussão}

Analisando as pacientes que procuraram nosso serviço com quadro de ameaça de abortamento no primeiro trimestre, foi diagnosticada a inviabilidade da gestação em $46,6 \%$ dos casos, por meio do exame ultra-sonográfico. Este dado é compativel com trabalhos anteriores apresentados na Tabela 3, cujo percentual variou entre 39 e $45 \%{ }^{6,7,10}$

Tabela 3 - Resultados de 3 investigações sobre achados ultra-sonográficos em porcentagens em gestantes que apresentaram quadro de ameaça de abortamento.

\begin{tabular}{|c|c|c|c|c|c|c|c|}
\hline \multirow{3}{*}{ Gestação viável } & \multicolumn{7}{|c|}{ Gestação Inviável } \\
\hline & \multicolumn{3}{|c|}{ Abortamento } & \multirow[t]{2}{*}{ Anembrionada } & \multirow[t]{2}{*}{ Ectópica } & \multirow{2}{*}{$\begin{array}{c}\text { Mola } \\
\text { hidatiforme }\end{array}$} & \multirow[t]{2}{*}{ Referências } \\
\hline & Incompleto & Completo & Retido & & & & \\
\hline 61,0 & 5,0 & - & 15,0 & 8,0 & 1,0 & 1,0 & 7 \\
\hline 55,6 & 8,8 & 0,9 & 7,3 & 14,4 & 12,8 & 0,2 & 10 \\
\hline
\end{tabular}

Os achados ultra-sonográficos nas gestações com ameaça de abortamento apresentam percentual variável nas diversas séries da literatura. Neste estudo, a incidência de abortamento incompleto foi de $19 \%$, que é maior do que o observado por outros autores: 5 a $8,8 \%{ }^{6,7,10}$. Com a gestação anembrionada a nossa incidência foi de $6,1 \%$, sendo menor que o mostrado em outros estudos cuja variação foi de 8 a $26 \%{ }^{6,7,10}$. Talvez estas diferenças sejam decorrentes do maior tempo transcorrido entre o início do sangramento e a realização do exame ultra-sonográfico. Deste modo, os casos de gestação anembrionada poderiam evoluir com eliminação parcial de seu conteúdo, apresentando no momento do exame o diagnóstico ultra-sonográfico de abortamento incompleto. $\mathrm{O}$ achado ultra-sonográfico de saco gestacional menor que o esperado é freqüente na avaliação de primeiro trimestre. Quando existe dúvida entre uma gestação anembrionada ou erro de data, devese proceder à reavaliação ultra-sonográfica no período de uma a duas semanas, para conclusão diagnóstica.

A incidência de aborto retido, mola hidatiforme e gestação ectópica foi similar à da maioria dos outros estudos. É descrito na literatura médica que alguns casos de mola hidatiforme no início da gestação podem apresentar vesículas pobremente desenvolvidas, tornando-se no exame ultra-sonográfico indistingüível de um aborto retido ${ }^{11}$. Esta situação foi vivenciada em um caso no presente estudo e o diagnóstico foi definido pelo resultado do exame anatomopatológico.

Estudos anteriores demonstraram que a duração do sangramento por período de um a dois dias correlacionava-se com percentual de abortamento entre 32 e $47 \%$. Quando o sangramento apresentava duração de três ou mais dias, o abortamento ocorria em 53 a $60 \%$ dos $\operatorname{casos}^{6,7}$. No presente estudo, também foi observada correlação entre o aumento de perdas fetais e o tempo de sangramento. Quando o sangramento ocorreu por periodo de um a dois dias, 30,9\% das gestantes abortaram; com três a sete dias, $47 \%$ abortaram, e com período de sangramento superior a sete dias, $80 \%$ das gestantes abortaram.

O antecedente obstétrico é apontado como importante fator contributivo para o risco de abortamento por Regan et al. ${ }^{12}$ Em seu trabalho o percentual de abortamentos foi de $19 \%$ em gestantes com antecedente de abortamento na última gestação, ao passo que, entre as gestantes primigestas ou com antecedente de parto bem sucedido na última gestação, o percentual foi de apenas 5\%. Entretanto, este percentual não tem sido confirmado por outros autores ${ }^{4,9,13}$, inclusive por este trabalho. Everett ${ }^{4}$ refere que mulheres com antecedente de abortamento não apresentam um risco significativamente maior de uma segunda perda fetal consecutiva. 
Stabile et al. ${ }^{10}$ referem que o risco de abortamento espontâneo aumenta com a idade materna. Mantoni ${ }^{7}$, e Regan et al. ${ }^{12}$ não identificaram diferença de idade materna entre as gestações que prosseguiram e as que abortaram. Neste estudo, quando a idade materna foi avaliada por faixas etárias, percentual similar de abortamento foi observado entre as gestantes até 30 anos $(46,6 \%)$, de 30 a 35 anos (45\%) e acima de 35 anos (48\%).

Existe uma tendência a aumento da taxa de abortamentos quando o sangramento vaginal ocorre em idade gestacional mais avançada ${ }^{6}$, porém, existe controvérsia quanto à influência da idade gestacional em que ocorreu o sangramento na taxa de abortamento. Mantoni ${ }^{7}$ relatou $35 \%$ de perdas fetais quando o sangramento ocorreu entre cinco e nove semanas de gestação e $45 \%$ de perdas quando o sangramento ocorreu entre 10 e 20 semanas de gestação. Jouppila et al. ${ }^{6}$ observaram que, quando o sangramento ocorreu entre cinco e nove semanas de gestação, $51 \%$ dos casos evoluíram para o abortamento, ao passo que entre $10 \mathrm{e}$ 14 semanas, 53\% apresentaram esta mesma evolução. No presente estudo, na ocorrência de sangramento vaginal entre seis e nove semanas, $44,2 \%$ das gestações foram inviáveis. Com sangramento entre 10 e 14 semanas a inviabilidade foi diagnosticada em $39,8 \%$ dos casos.

A literatura carece de estudos demonstrando o benefício do repouso nos casos com ameaça de abortamento no primeiro trimestre da gravidez; entretanto, a recomendação de repouso até que cesse o sangramento é uma conduta comum no meio médico.

Muitas vezes as gestantes que apresentam sangramento vaginal percorrem várias maternidades na expectativa de um tratamento que cesse o sangramento, ou mesmo à procura de um exame de ultra-sonografia para elucidação diagnóstica. Uma vez que o exame ultra-sonográfico é capaz de diagnosticar a inviabilidade da gestação e esta situação ocorre em aproximadamente metade destes casos, justifica-se a indicação deste exame para investigação primária de qualquer gestante que apresente quadro de ameaça de abortamento. Entretanto, apenas uma pequena parcela deste contingente de gestantes consegue realizar o exame ultra-sonográfico no sistema público de saúde, dada a insuficiente disponibilidade deste exame em face da grande demanda. A maioria dos serviços em nosso país adotam a conduta expectante, aguardando a possibilidade de ocorrência do abortamento espontâneo. Nos casos em que o exame ultra-sonográfico constata embrião ou feto vivo, na maioria das vezes não é revelada a causa do sangramento, mas certamente contribui para diminuir a ansiedade dos pais.

\section{SUMMARY}

Objective: to evaluate ultrasound findings in pregnant women with threatened abortion in the first trimester of pregnancy. Methods: transabdominal and transvaginal ultrasound scans were performed in patients with vaginal bleeding with previous positive pregnancy test. Patients with 6-14-week gestation (by the last menstrual period or ultrasound scan), with closed cervix on clinical evaluation were included. Multiple pregnancies and those patients who have tried abortion by using abortive drugs or manipulation were excluded.

Results: in 132 of 247 (53.4\%) the pregnancy was viable and in $46.6 \%$ (115/247) the pregnancy was nonviable. Incomplete miscarriage was found in 19\% (47/247), complete miscarriage in $8.5 \%$ (21/247), missed abortion in $7.7 \%$ (19/ 247), anembryonic pregnancy in $6.1 \%$ (15/247), ectopic pregnancy in $4.5 \%(11 / 247)$ and hydatidiform mole in $0.8 \%$ (2/247).

Conclusion: almost half (46.6\%) of the pregnancies with threatened abortion in the first trimester were diagnosed as a nonviable pregnancy. The ultrasound scan can help to define this condition and the management of the pregnancy.

KEY WORDS: Threatened abortion. Ultrasound scan. First trimester pregnancy. Hydatidiform mole.

\section{Agradecimentos}

À FAPESP pela permissão para utilização do aparelho de ultra-som cedido ao projeto No. 97/ 5581-7.

\section{Referências}

1. South J, Naldrett J. The effect of vaginal bleeding in early pregnancy on the infant born after the $28^{\text {th }}$ week of pregnancy. J Obstet Gynaecol $\mathrm{Br}$ Commonw 1973; 80: 236-41.

2. Hertz JB. Diagnosis procedures in threatened abortion. Obstet Gynecol 1984; 64: 223-9.

3. Vaginal bleeding in early pregnancy. BMJ 1980; 281:470.

4. Everett C. Incidence and outcome of bleeding before the 20th week of pregnancy: prospective study from general practice. BMJ 1997; 315:32-4.

5. Johannsen, A. The prognosis of threatened abortion. Acta Obstet Gynecol Scand 1970; 49:89-93.

6. Jouppila P. Clinical and ultrasonic aspects in the diagnosis and follow-up of patients with early pregnancy failure. Acta Obstet Gynecol Scand 1980; 59:405-9. 
7. Mantoni M. Ultrasound signs in threatened abortion and their prognostic significance. Obstet Gynecol $1985 ; 65: 471-5$

8. Cashner KA, Christopher CR, Dysert GA. Spontaneous fetal loss after demonstration of a live fetus in the first trimester. Obstet Gynecol 1987; 70:827-30.

9. Hill LM, Guzick D, Fries J, Hixson J. Fetal loss rate after ultrasonically documented cardiac activity between 6 and 14 weeks menstrual age. J Clin Ultrasound 1991; 19: 221-3.

10.Stabile I, Campbell S, Grudzinskas JG. Ultrasonic assessment of complications during the first trimester of pregnancy. Lancet 1987; 2:1237-40.
11.Coleman BG, Arger PH. Ultrasound in early pregnancy complications. Clin Obstet Gynecol $1988 ; 31: 3-18$.

12.Regan L, Braude PR, Trembath PL. Influence of past reproductive performance on risk of spontaneous abortion. BMJ 1989; 299:541-5.

13. Cohen-Overbeek TE, Hop WC, den Ouden M, Pijpers L, Jahoda MG, Wladimiroff JW. Spontaneous abortion rate and advanced maternal age: consequences for prenatal diagnosis. Lancet 1990; 336:27-9. 